\title{
Water vapor transport to a semi-infinite material with simultaneous varying surface relative humidity and temperature
}

\author{
Carl-Eric Hagentoft* \\ Building Technology, Chalmers University of Technology, SE-412 96 Göteborg, Sweden
}

\begin{abstract}
The water vapor transfer between the indoor air and hygroscopic finishing materials is of importance for the moisture balance of the room. Most protocols for determining the effect are based on isothermal conditions and cycling relative humidity in the form of square wave or sinusoidal functions. A new analytical solution for a material exposed to a both time varying surface relative humidity and temperature is presented in the paper. The time varying temperature inside the material is assumed to follow the surface temperature throughout the material layer since the reaction time for temperature changes in a reasonable thin surface material is rather short compared with the one for moisture changes. The semiinfinite approach is justified by the fact that the penetration depth for moisture variations are very limited for diurnal variations. The analytical approach and solution are presented in the paper
\end{abstract}

\section{Introduction}

Moisture uptake in the interior surface materials due to varying humidity in the indoor air is of importance both for the humidity levels of the room itself and for the moisture conditions in the surface materials. There have been research on this topic [1-3] and standards [4] and some ongoing measurements [5].

However, there has been less focus on cases with both a varying RH and temperature. Some recent results on this are presented in [6-7]. This study gives new handy analytical expressions for the effect of combined variations in $\mathrm{RH}$ and temperature. In parallel to this paper, an experimental validation of the model is under way.

\section{Governing equations}

In this study the surface vapor resistance is neglected which means that the surface RH is always equal to the room RH. This will represent the case of maximum interaction.

\subsection{Equations}

The moisture balance equation reads:

$$
-\frac{\partial}{\partial x}\left(-\delta_{v} \frac{\partial v}{\partial x}\right)=\frac{\partial w}{\partial t}
$$

Here, $v\left(\mathrm{~kg} / \mathrm{m}^{3}\right)$ is the humidity by volume and $w\left(\mathrm{~kg} / \mathrm{m}^{3}\right)$ is the moisture content.
The analysis in this paper assumes that the temperature of the surface material always follows the interior temperature without any delay. This is a reasonable assumption since temperature changes are much more rapid than moisture changes and that it is only the thin interior surface layer that is affected by variations in indoor cyclic moisture variations.

\subsection{Simplified equations}

Two simplifications will be introduced. The first one is that the vapor diffusion coefficient $\delta_{v}\left(\mathrm{~m}^{2} / \mathrm{s}\right)$ is constant:

$$
\delta_{v}=\delta_{v}^{0}
$$

The second simplification is that the slope of the sorption curve is constant. Furthermore, hysteresis is neglected.

$$
\frac{\partial w}{\partial \varphi}=\xi
$$

The moisture balance equation then becomes:

$$
\delta_{v}^{0} \frac{\partial^{2} v}{\partial x^{2}}=\delta_{v}^{0} v_{s}(T) \frac{\partial^{2} \varphi}{\partial x^{2}}=\frac{\partial w}{\partial t}=\frac{\partial w}{\partial \varphi} \frac{\partial \varphi}{\partial t}=\xi \frac{\partial \varphi}{\partial t}
$$

Here, $v_{s}$ is the humidity by volume at saturation. Introducing the vapor moisture diffusivity $a_{v}\left(\mathrm{~m}^{2} / \mathrm{s}\right)$ :

$$
\frac{\partial^{2} \varphi}{\partial x^{2}}=\frac{1}{a_{v}} \frac{\partial \varphi}{\partial t} \quad a_{v}=\frac{\delta_{v}^{0} v_{s}(T)}{\xi}
$$




\section{Two basic solutions for constant temperature and zero surface resistance}

\subsection{Step change}

A material surface layer with initial relative humidity of $\varphi_{0}$ and temperature of $T_{0}$ is considered.

We have a RH step change at the boundary, $x=0$, at time zero. The surface $\mathrm{RH}$ is:

$$
\varphi_{s}=\varphi_{0}+\Delta \varphi
$$

The analytical solution [1] for a semi-infinite domain $x \geq 0, t \geq 0$ is:

$$
\varphi(x, t)=\varphi_{0}+\Delta \varphi \cdot \operatorname{erfc}\left(\frac{x}{\sqrt{4 a_{v} t}}\right)
$$

Here, erfc is the complimentary error function. The penetration depth, i.e. the depth were we approximately find half the disturbance of what happened at the boundary has propagated is:

$$
x_{0.5}=\sqrt{a_{v} t}
$$

Example: For wood this depth is around $0.0005 \mathrm{~m}$ after $1 \mathrm{~h}, 0.0007 \mathrm{~m}$ after $2 \mathrm{~h}, 0.003 \mathrm{~m}$ after 1 day and $0.007 \mathrm{~m}$ after a week.

\subsection{Periodic change}

If we have the following variation of the $\mathrm{RH}$ at the material surface, $x=0$ :

$$
\varphi_{s}=\varphi_{0}+\varphi_{A} \cdot \cos \left(\frac{2 \pi t}{t_{p}}\right)
$$

Here, $t_{p}$ (s) is the time period of the cosinusoidal variation. For the steady periodic case, the following well-known expression [1] for a semi-infinite domain $x \geq 0$ reads:

$$
\varphi(x, t)=\varphi_{0}+\varphi_{A} \cdot e^{-x / d_{p v}} \cos \left(\frac{2 \pi t}{t_{p}}-\frac{x}{d_{p v}}\right)
$$

The periodic penetration depth $d_{p v}(\mathrm{~m})$, i.e. the depth were the amplitude of the RH has diminished with a factor $e^{-1} \approx 0.37$ is:

$$
d_{p v}=\sqrt{\frac{a_{v} t_{p}}{\pi}}
$$

Example: For wood this depth is around 0.001-0.002 m for a diurnal variation $\left(t_{p}=24 \mathrm{~h}\right)$ and $0.03 \mathrm{~m}$ for a yearly one.

These numbers show that the penetration depth of moisture in to the material is very small for diurnal variations.
This periodic variation is of particular interest in this paper. The moisture flow $g\left(\mathrm{~kg} / \mathrm{m}^{2} / \mathrm{s}\right)$ into the material at $\mathrm{x}=0$ becomes:

$$
\begin{aligned}
& g=g_{A} \cdot \cos \left(\frac{2 \pi t}{t_{p}}+\frac{\pi}{4}\right) \\
& g_{A}=\varphi_{A} \frac{\sqrt{2 \pi a_{v}}}{\sqrt{t_{p}}} \xi \\
& \left(g_{A}=\varphi_{A} d_{p v} \xi \sqrt{2} \frac{\pi}{t_{p}}\right)
\end{aligned}
$$

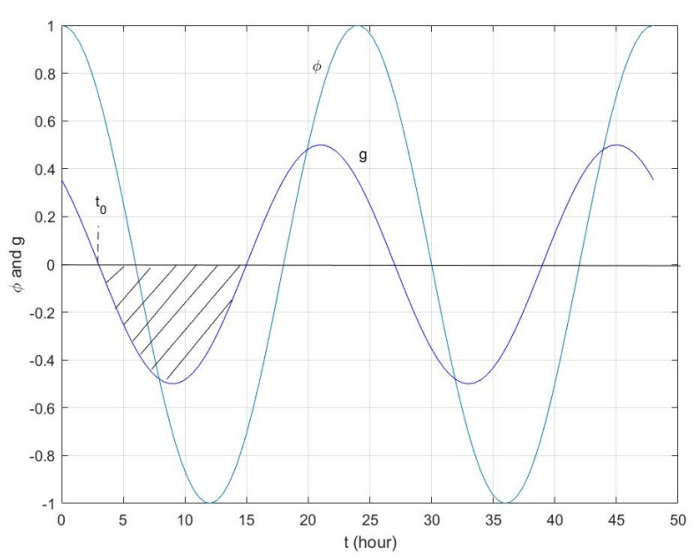

Fig 1. Principle sketch of the diurnal boundary $\mathrm{RH}$ variation (9) and the moisture flow into the material (12). The total moisture uptake during a half period, $m_{A}$, is indicated by the hatched area. The time $t_{0}$ is also marked.

We are interested in the total uptake of moisture by the surface during a half period. The magnitude of the moisture uptake $m_{A}\left(\mathrm{~kg} / \mathrm{m}^{2}\right)$ from time $t_{0}$ to time $t_{0}+t_{p} / 2$ is:

$$
m_{A}=\left|\int_{t_{0}}^{t_{0}+t_{p} / 2} g\left(t^{\prime}\right) d t^{\prime}\right|
$$

The time $t_{0}$ is chosen so that the net uptake of moisture is zero at this time, see Fig 1. For the periodic $\mathrm{RH}$ variation according to (9) and (12) it is equal to $t_{p} / 8$.

For steady periodic variations the net accumulation of moisture in the material is on average zero over time. The amplitude of the integrated mass flow $\left(\mathrm{kg} / \mathrm{m}^{2}\right),(13)$, into and out from the material surface during a half period, [1]:

$$
\begin{aligned}
& m_{A}=g_{A} \cdot \frac{t_{p}}{\pi}=\varphi_{A} d_{p v} \xi \sqrt{2} \\
& m_{A}=\frac{\varphi_{A}}{2 \sqrt{\pi}} \xi \sqrt{a_{v} t_{p}} \cdot f_{A} \quad f_{A}=2 \sqrt{2}
\end{aligned}
$$




\section{Varying temperature}

We will consider the case of time varying temperature:

$$
\frac{\partial^{2} \varphi}{\partial x^{2}}=\frac{1}{a_{v}(t)} \frac{\partial \varphi}{\partial t} \quad a_{v}(t)=\frac{\delta_{v}^{0} v_{s}(T(t))}{\xi}
$$

Here, the material temperature is equal to the boundary temperature. It is purely a function of time:

$$
\frac{\partial^{2} \varphi}{\partial x^{2}}=\frac{1}{a_{v}(t)} \frac{\partial \varphi}{\partial t}
$$

A variable substitution is introduced:

$$
\tau(t)=\int_{0}^{t} a_{v}\left(t^{\prime}\right) d t^{\prime}
$$

The moisture balance equation is transformed to:

$$
\frac{\partial^{2} \varphi}{\partial x^{2}}=\frac{\partial \varphi}{\partial \tau}
$$

Here, we have an equation that is similar to the onedimensional moisture balance equation with the diffusivity $a_{v}$ equal to 1 . The equation is linear when using this transformed time variable and superposition techniques can be used. We therefore only need the basic solution for a unit-step change or sinusoidal variations to handle complex time-wise changes in the RH. For a unit step change we for instance have:

$$
\left\{\begin{array}{l}
\varphi(x, \tau)=\varphi_{0}+\Delta \varphi \cdot \operatorname{erfc}\left(\frac{x}{\sqrt{4 \tau}}\right) \\
x \geq 0, \tau \geq 0
\end{array}\right.
$$

And for a cosinusoidal variation (steady-periodic) in the $\tau$ regime with the time period $\tau_{p}$ :

$$
\begin{gathered}
\varphi(x, \tau)=\varphi_{0}+\varphi_{A} \cdot e^{-x / d_{p v}} \cos \left(\frac{2 \pi \tau}{\tau_{p}}-\frac{x}{d_{p v}}\right) \\
x \geq 0 \quad d_{p v}=\sqrt{\frac{\tau_{p}}{\pi}}
\end{gathered}
$$

\section{Wave train}

\subsection{RH in the material layer}

Assume a periodic square shaped boundary RH and temperature in time (corresponding to a wave train symmetrical around $t=0$ ) with the time period $t_{p}$. For simplicity we choose that half of the time $\mathrm{RH}$ has its highest value, $\varphi_{0}+\varphi_{A}$, and temperature $T_{l}$ and the other half of the time the lowest one, $\varphi_{0}-\varphi_{A}$, and temperature $T_{2}$. Alternative proportions can be studied using the same procedure as below.

$$
\begin{aligned}
& \varphi_{s}=\left\{\begin{array}{lc}
\varphi_{0}+\varphi_{A} & 0 \leq t \leq \frac{t_{p}}{4} \quad \frac{3 t_{p}}{4} \leq t \leq t_{p} \\
\varphi_{0}-\varphi_{A} & \frac{t_{p}}{4} \leq t \leq \frac{3 t_{p}}{4}
\end{array}\right. \\
& T_{s}=\left\{\begin{array}{ccc}
T_{1} & \left(a_{v}=a_{v 1}\right) & 0 \leq t \leq \frac{t_{p}}{4} \quad \frac{3 t_{p}}{4} \leq t \leq t_{p} \\
T_{2} & \left(a_{v}=a_{v 2}\right) & \frac{t_{p}}{4} \leq t \leq \frac{3 t_{p}}{4}
\end{array}\right.
\end{aligned}
$$

When transformed to the $\tau$-variable we get the high $\mathrm{RH}$ value $\varphi_{0}+\varphi_{A}$ and temperature $T_{1}$ for the following intervals:

$$
0 \leq \tau \leq \tau\left(\frac{t_{p}}{4}\right) \quad \tau\left(\frac{3 t_{p}}{4}\right) \leq \tau \leq \tau\left(t_{p}\right)
$$

, and low RH value $\varphi_{0}-\varphi_{A}$ and temperature $T_{2}$ for:

$$
\tau\left(\frac{t_{p}}{4}\right) \leq \tau \leq \tau\left(\frac{3 t_{p}}{4}\right)
$$

From the definition of $\tau$ (17):

$$
\begin{aligned}
& \tau\left(\frac{t_{p}}{4}\right)=\frac{a_{v 1} \cdot t_{p}}{4} \\
& \tau\left(\frac{3 t_{p}}{4}\right)=\frac{a_{v 1} \cdot t_{p}}{4}+\frac{a_{v 2} \cdot t_{p}}{2} \\
& \tau_{p}=\tau\left(t_{p}\right)=\frac{a_{v 1}+a_{v 2}}{2} t_{p}=\bar{a}_{v} \cdot t_{p}
\end{aligned}
$$

Figure 1 shows the boundary values as functions of $t$ and $\tau$. 

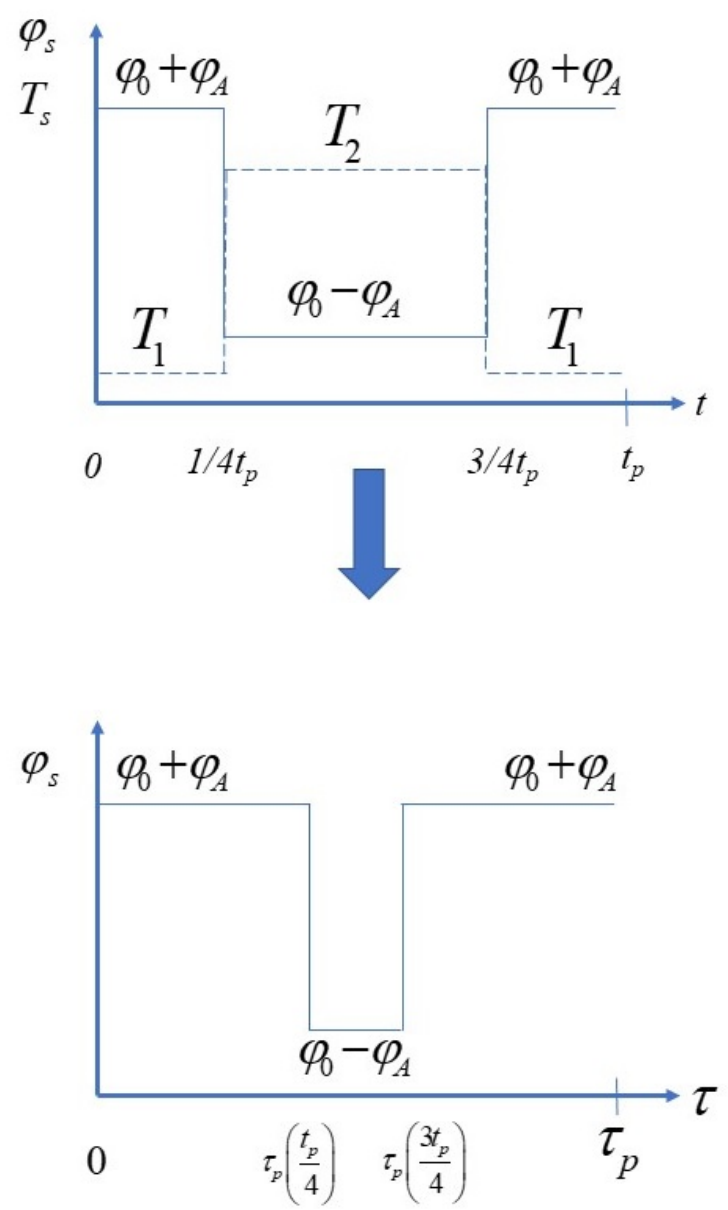

Fig 2. Boundary $\mathrm{RH}$ and temperature as functions of $t$ and $\tau$.

The fraction, $\alpha$, of the time period $\tau_{p}$ when the $\mathrm{RH}$ has its highest value (and $a_{v}=a_{v 1}$ ) is:

$$
\alpha=\frac{a_{v 1}}{a_{v 1}+a_{v 2}} \quad\left(=\frac{a_{v 1} \cdot t_{p} / 2}{\left(a_{v 1}+a_{v 2}\right) \cdot t_{p} / 2}\right)
$$

Using Fourier series in $\tau$ regime, the wave train can be written as:

$$
\begin{aligned}
& \varphi_{s}=\varphi_{0}+(2 \alpha-1) \cdot \varphi_{A}+ \\
& +\frac{4 \varphi_{A}}{\pi} \sum_{n=1}^{\infty} \frac{\sin (n \pi \alpha)}{n} \cos \left(2 n \pi \frac{\tau(t)}{\tau_{p}}\right)
\end{aligned}
$$

When $\alpha=0.5$, the high and low values of $a_{v}$ are equal and the second term will vanish. This represent the isothermal ( $a_{v 1}=a_{v 2}$ ). For other values of $\alpha$ there will be a change in the average value of RH in the material from $\varphi_{0}$.

Since we know the solution for the heat conduction equation for cosinusoidal boundary conditions (20) we get:

$$
\begin{aligned}
& \varphi(x, t)=\varphi_{0}+(2 \alpha-1) \cdot \varphi_{A}+ \\
& +\frac{4 \varphi_{A}}{\pi} \sum_{n=1}^{\infty} \frac{\sin (n \pi \alpha)}{n} e^{-x / d_{p v, n}} \cos \left(2 n \pi \frac{\tau(t)}{\tau_{p}}-\frac{x}{d_{p v, n}}\right) \\
& d_{p v, n}=\sqrt{\frac{\tau_{p}}{n \pi}}
\end{aligned}
$$

For the isothermal case $\left(a_{v 1}=a_{v 2}\right)$

$$
\begin{aligned}
& \varphi(x, t)=\varphi_{0}+ \\
& +\frac{4 \varphi_{A}}{\pi} \sum_{n=1}^{\infty} \frac{\sin (n \pi / 2)}{n} e^{-x / d_{p v, n}} \cos \left(2 n \pi \frac{t}{t_{p}}-\frac{x}{d_{p v, n}}\right) \\
& d_{p v, n}=\sqrt{\frac{a_{v} t_{p}}{n \pi}}
\end{aligned}
$$

Figure 3 shows the relative amplitude of $\mathrm{RH}$ and the average $\mathrm{RH}$ as a function of depth from the surface using (27) for a specific case.

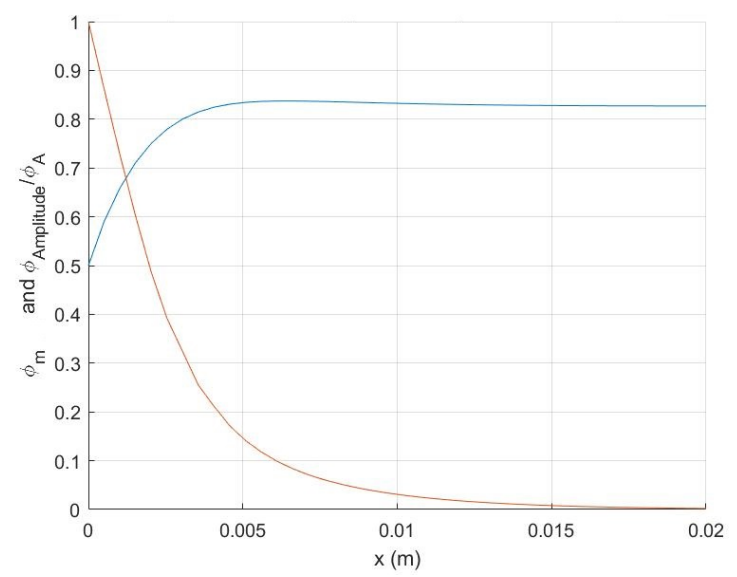

Fig 3. Example of the relative amplitude of $\mathrm{RH}$ and the average $\mathrm{RH}$ as a function of depth from the surface.

$\varphi_{0}=0.5 \varphi_{A}=0.4 a_{v 1}=10 \cdot 10^{-10} \mathrm{~m}^{2} / \mathrm{s} a_{v 2}=1 \cdot 10^{-10} \mathrm{~m}^{2} / \mathrm{s}$

\subsection{Moisture uptake}

The moisture flow $g\left(\mathrm{~kg} / \mathrm{m}^{2} / \mathrm{s}\right)$ into the material is:

$$
\begin{aligned}
& g(t)=-\left.\delta_{v}^{0} v_{s}(T(t)) \frac{\partial \varphi(x, t)}{\partial x}\right|_{x=0}= \\
& =\varphi_{A} \delta_{v}^{0} v_{s}(T(t)) \sqrt{2} \frac{4}{\pi} \sum_{n=1}^{\infty} \frac{\sin (n \pi \alpha)}{n \cdot d_{p v, n}} \cos \left(2 n \pi \frac{\tau(t)}{\tau_{p}}+\frac{\pi}{4}\right)= \\
& =\varphi_{A} \sqrt{2 \pi} \xi \frac{a_{v}(t)}{\sqrt{\tau_{p}}} \frac{4}{\pi} \sum_{n=1}^{\infty} \frac{\sin (n \pi \alpha)}{\sqrt{n}} \cos \left(2 n \pi \frac{\tau(t)}{\tau_{p}}+\frac{\pi}{4}\right)=
\end{aligned}
$$

For the isothermal case ( $\alpha=1 / 2)$ :

$$
\begin{aligned}
& g(t)=\varphi_{A} \sqrt{2 \pi} \xi \sqrt{a_{v}} \cdot \frac{1}{\sqrt{t_{p}}} \\
& \cdot \frac{4}{\pi} \sum_{m=0}^{\infty} \frac{(-1)^{m}}{\sqrt{2 m+1}} \cos \left(2 \cdot(2 m+1) \pi \frac{t}{t_{p}}+\frac{\pi}{4}\right)
\end{aligned}
$$


The integrated $m\left(\mathrm{~kg} / \mathrm{m}^{2}\right)$ moisture uptake from time zero to time $t$ is:

$$
m(t)=\int_{0}^{t} g\left(t^{\prime}\right) d t^{\prime}
$$

The formula can be reformulated using a variable substitution, $s=\tau(t)$ :

$$
\begin{aligned}
& m(\tau(t))=\frac{\varphi_{A}}{2 \sqrt{\pi}} \xi \sqrt{\tau_{p}} \cdot f_{m} \\
& f_{m}= \\
& =\frac{4}{\pi} \sum_{n=1}^{\infty} \frac{\sin (n \pi \alpha)}{n \sqrt{n}}\left[\sqrt{2} \cdot \sin \left(2 n \pi \frac{\tau(t)}{\tau_{p}}+\frac{\pi}{4}\right)-1\right]
\end{aligned}
$$

Figure 4 shows $f_{m}$, (32), for a case. As seen from the figure the moisture is leaving the material at time $t_{0}$ until time $t_{0}+t_{p} / 2$ when the moisture uptake is increasing again. The total moisture uptake/release during a half cycle is equal $m_{A}$, which is illustrated in Fig 4.

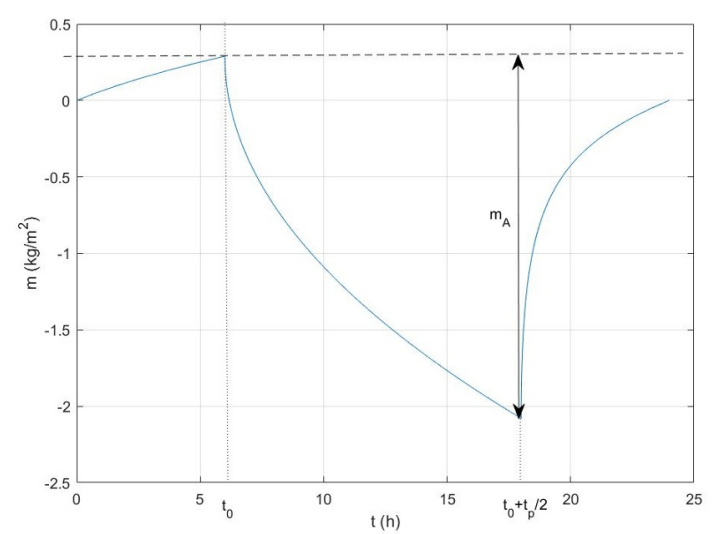

Fig 4. Parameter $f_{m}$, (32), for the integrated moisture uptake as a function of time $t$ during a day. Diurnal variations $\left(t_{p}=24 \mathrm{~h}\right)$.

$a_{v 1}=10 \cdot 10^{-10} \mathrm{~m}^{2} / \mathrm{s} \quad a_{v 2}=1 \cdot 10^{-10} \mathrm{~m}^{2} / \mathrm{s}$

The moisture uptake during a half period determined by (13) and (31-32) becomes:

$$
m_{A}=\frac{\varphi_{A}}{2 \sqrt{\pi}} \xi \sqrt{\tau_{p}} \cdot f_{A}
$$

The time lag $t_{0}$ and the amplitude function $f_{A}$ is practically found by integrating the moisture flow over time $m(t)$, (31). The amplitude $m_{A}$ is then obtained from the difference between $m_{\max }$ and $m_{\min }$. See Fig 4 . For the studied case the time $t_{0}$ is approximately equal to $t_{p} / 4$, i.e the time when RH goes from a high to a low value.

The amplitude parameter $f_{A}$ of the integrated mass flow into and out from the material surface (33) is given in Table 1.
Table 1. The amplitude parameter $f_{A}$ for the determination of the total moisture uptake during a half cycle.

\begin{tabular}{|c|c|}
\hline$\alpha$ & $f_{A}(\alpha)$ \\
\hline 0 & 0 \\
\hline 0.0001 & 0.0802 \\
\hline 0.001 & 0.2541 \\
\hline 0.01 & 0.8034 \\
\hline 0.05 & 1.777 \\
\hline 0.10 & 2.479 \\
\hline 0.15 & 2.982 \\
\hline 0.20 & 3.369 \\
\hline 0.25 & 3.672 \\
\hline 0.30 & 3.907 \\
\hline 0.35 & 4.083 \\
\hline 0.40 & 4.205 \\
\hline 0.45 & 4.277 \\
\hline 0.50 & 4.300 \\
\hline
\end{tabular}

Due to symmetry we will get the same amplitude of the integrated mass flow in to the material if let $a_{v l}$ occur at the high boundary RH and $a_{v 2}$ at the low one:

$$
f_{A}(\alpha)=f_{A}(1-\alpha)
$$




\subsection{Example}

The material surface is made of spruce:

$\delta_{v}^{0}=1.5 \cdot 10^{-6} \mathrm{~m}^{2} / \mathrm{s} \quad \xi=100 \mathrm{~kg} / \mathrm{m}^{3}$

The RH will be high when the temperature is low and low when the temperature is high. The following boundary values are assumed.

$\varphi_{0}=0.4 \quad \varphi_{A}=0.3 \quad t_{p}=24 \cdot 3600 \mathrm{~s}$

$T_{1}=18^{\circ} \mathrm{C} \quad T_{2}=25^{\circ} \mathrm{C}$

We get the following vapor diffusivities:

$$
\begin{aligned}
& a_{v 1}=\frac{\delta_{v}^{0} v_{s}\left(T_{1}\right)}{\xi}=\frac{1.5 \cdot 10^{-6} \cdot v_{s}(18)}{100}=2.304 \cdot 10^{-10} \\
& a_{v 2}=\frac{\delta_{v}^{0} v_{s}\left(T_{2}\right)}{\xi}=\frac{1.5 \cdot 10^{-6} \cdot v_{s}(25)}{100}=3.455 \cdot 10^{-10}
\end{aligned}
$$

The $\alpha$ parameter (25) becomes:

$$
\begin{aligned}
& \alpha=\frac{a_{v 1}}{a_{v 1}+a_{v 2}}=\frac{2.304}{2.304+3.455}=0.4 \\
& \bar{\varphi}=\varphi_{0}+(2 \alpha-1) \cdot \varphi_{A}=0.34
\end{aligned}
$$

The average $\mathrm{RH}$ in the material will be lower than the average of the boundary due to changes of the temperature over time.

The magnitude of the integrated mass flow into and out from the material surface is:

$$
\begin{aligned}
& m_{A}=\frac{0.3}{2 \sqrt{\pi}} 100 \sqrt{\frac{2.304+3.455}{2} 10^{-10} \cdot 24 \cdot 3600} \cdot f_{A}(0.4) \\
& m_{A}=0.0422 \cdot 4.205=0.178 \mathrm{~kg} / \mathrm{m}^{2}
\end{aligned}
$$

\section{Conclusions}

Explicit analytical formulas for the moisture distribution inside a material that is exposed to cyclic variations in $\mathrm{RH}$ and temperature are derived. The boundary $\mathrm{RH}$ and temperature have different values during each half period. The formula can be used to estimate the uptake and release of moisture to an exposed material surface as long as the periodic penetration depth is much smaller than the thickness of the material.

\section{References}

1. C-E. Hagentoft, 2001. Introduction to building Physics. Studentlitteratur AB, Lund Sweden.

2. Kaisa Svennberg 2006. Doctoral thesis: Moisture Buffering in the Indoor Environment. Lund University TVBH-06-1016.

3. R. Peuhkuri \& C. Rode. 2005. Moisture buffer value: Analytical determination and use of dynamic measurements. Nordic Building Physics Symposium

4. C. Rode, R. Peuhkuri, L.H. Mortensen, K. Hansens, B. Time, A. Gustavsen, T. Ojanen, J, Ahonen, K, Svennberg, L.E. Harderup, J. Arfvidsson J, Ojanen T. NORDTEST Project on Moisture Buffer Value of Materials. Technical University of Denmark (2005)

5. Valeria Cascione et.al. 2019. The effect of air velocity on moisture buffering. CESBP, Prague.

6. Bylund Melin C., 2018. Wooden Objects in Historic Buildings: Effects of Dynamic Relative Humidity and Temperature. Ph.D. Thesis, University of Gothenburg, Gothenburg, Sweden.

7. Charlotta Bylund Melin et.al. 2018. Simulations of Moisture Gradients in Wood Subjected to Changes in Relative Humidity and Temperature Due to Climate Change. Journal of Geosciences, 2018, 8, 378 